\title{
Inhibition of SHP2 leads to mesenchymal to epithelial transition in breast cancer cells
}

\author{
X-D Zhou' and YM Agazie ${ }^{\star, 1,2}$
}

The Src homology phosphotyrosyl phosphatase 2 (SHP2) is an essential transducer of mitogenic and cell survival signaling in the epidermal growth factor receptor (EGFR) signaling pathway. However, the role of SHP2 in aberrant EGFR and human EGFR2 (HER2) signaling and cancer, particularly in breast cancer, has not been investigated. Here, we report that SHP2 is required for mitogenic and cell survival signaling and for sustaining the transformation phenotypes of breast cancer cell lines that overexpress EGFR and HER2. Inhibition of SHP2 suppressed EGF-induced activation of the Ras-ERK and the phosphatidylinositol 3 kinase-Akt signaling pathways, abolished anchorage-independent growth, induced epithelial cell morphology and led to reversion to a normal breast epithelial phenotype. Furthermore, inhibition of SHP2 led to upregulation of E-cadherin (epithelial marker) and downregulation of fibronectin and vimentin (mesenchymal markers). These results indicate that SHP2 promotes breast cancer cell phenotypes by positively modulating mitogenic and cell survival signaling, by suppressing E-cadherin expression which is known to play a tumor suppressor role and by sustaining the mesenchymal state as evidenced by the positive impact on fibronectin and vimentin expression. Therefore, SHP2 promotes epithelial to mesenchymal transition, whereas its inhibition leads to mesenchymal to epithelial transition. On the basis of these premises, we propose that interference with SHP2 function might help treat breast cancer.

Cell Death and Differentiation (2008) 15, 988-996; doi:10.1038/cdd.2008.54; published online 18 April 2008

Accumulating evidence suggests that the Src homology phosphotyrosyl phosphatase 2 (SHP2) could be important in cancer. First, SHP2 is essential for cell transformation induced by $\mathrm{v}-\mathrm{Src}^{1}$ and the constitutively active form of fibroblast growth factor receptor 3 (K650E-FGFR3). ${ }^{2,3}$ And second, SHP2 has been implicated in the development of Noonan syndrome and associated leukemia. ${ }^{4}$ This was based on the discovery of activating SHP2 mutations, sometimes referred to as leukemogenic SHP2 mutants, in patients with these diseases. ${ }^{4}$ In support of these discoveries, transgenic mice expressing leukemogenic SHP2 mutants develop lymphoid hyperplasia. ${ }^{5,6}$ Thus, SHP2 plays a significant role in cancer.

Earlier studies have shown that SHP2 plays a positive role in receptor tyrosine kinase (RTK) signaling. ${ }^{7}$ Particularly, SHP2 mediates RTK-induced activation of the Ras-extracellular signal-regulated kinase 1 and 2 (ERK1/2) and the phosphatidylinositol 3 kinase-Akt (also known as protein kinase B) signaling pathways. ${ }^{7,8}$ In the epidermal growth factor receptor (EGFR) signaling pathway, SHP2 promotes Ras activation by blocking the Ras GTPase-activating protein (RasGAP)-induced downregulation of Ras, via dephosphorylation of a docking site on the EGFR, Tyr992. ${ }^{9}$ Similarly, the Drosophila ortholog of SHP2, Corkscrew, transduces the
Torso RTK signaling by blocking Ras inactivation. ${ }^{10}$ Other reports also show that SHP2 dephosphorylates a RasGAPbinding site on the adapter protein Gab1 $1{ }^{11}$ leading to the same conclusion that SHP2 promotes Ras activation by blocking RasGAP. Furthermore, SHP2 promotes Ras and ERK $1 / 2$ activation by facilitating RTK-induced activation of the Src family kinases (SFKs). ${ }^{12}$

As its acronym indicates, SHP2 possesses two tandemly arranged Src homology $2(\mathrm{SH} 2)$ domains in the $\mathrm{N}$-terminal region, a phosphotyrosyl phosphatase (PTP) domain in the $\mathrm{C}$-terminal region ${ }^{7,8}$ and two tyrosine phosphorylation sites and a proline-rich motif in the extreme C-terminal region. The $\mathrm{SH} 2$ domains and the PTP domain are essential for the biological activity of SHP2, ${ }^{13}$ but its Tyr phosphorylation seems to be dispensable. ${ }^{14}$ In a resting state, SHP2 assumes a 'closed conformation' due to interaction between the $\mathrm{N}$-terminal SH2 and the PTP domains. The binding of the $\mathrm{SH} 2$ domains to phosphotyrosine-bearing proteins induces an open conformation and enzyme activation. ${ }^{15}$ Asp61 and Glu76 of the N-SH2 domain have been shown to be the mediators of the SH2-PTP domain interaction. Mutation of these residues to Ala activates SHP2, and mutations of these residues are suggested to be the causes of Noonan Syndrome and associated leukemia. ${ }^{4}$

\footnotetext{
${ }^{1}$ Department of Biochemistry and Molecular Pharmacology, West Virginia University, Morgantown, WV, USA and ${ }^{2}$ Marry Babb Randolph Cancer Center, West Virginia University, Morgantown, WV, USA

*Corresponding author: YM Agazie, Department of Biochemistry and Molecular Pharmacology and Marry Babb Randolph Cancer Center, West Virginia University, Morgantown, WV 26506, USA.

Tel: 304293 7756; Fax: 304293 6486;

E-mail: yagazie@hsc.wvu.edu

Keywords: breast cancer; EGFR; HER2; SHP2; transformation

Abbreviations: EGFR, epidermal growth factor receptor; EMT, epithelial to mesenchymal transition; HER2, human EGFR2; MET, mesenchymal to epithelial transition; PTP, phosphotyrosyl phosphatase; RTK, receptor tyrosine kinase; SH2, Src homology 2; SHP2, Src homology phosphotyrosyl phosphatase 2; shRNA, small hairpin RNA

Received 03.12.07; revised 14.3.08; accepted 18.3.08; Edited by R De Maria; published online 18.4.08
} 
We have recently reported that the SHP2 protein is elevated in breast cancer cell lines and overexpressed in primary breast tumors. ${ }^{16}$ However, the significance of SHP2 overexpression in breast cancer has not been determined. In this report, we show that SHP2 is required for EGF-induced signaling and for maintenance of transformation phenotypes in breast cancer cell lines that overexpress EGFR and human EGFR2 (HER2). In addition, we show that SHP2 is essential for sustaining the mesenchymal state of breast cancer cells, whereas its inhibition leads to mesenchymal to epithelial transition (MET) and reversion to a normal breast epithelial phenotype.

\section{Results}

Our recent report has shown that the SHP2 protein is elevated in a heterogeneous group of breast cancer cell lines. ${ }^{16}$ Hence, inhibition of SHP2 by dominant-negative expression in these cells might be difficult although not impossible. In addition, the dominant-negative strategy might provide inconclusive results due to insufficient inhibition. Therefore, we employed small hairpin RNA (shRNA)-mediated ablation of the SHP2 protein to fully appreciate the significance of SHP2 inhibition on RTK-induced signaling and transformation. In the current report, we have investigated the consequence of SHP2 inhibition in breast cancer cell transformation. The BT20 and the MDA-MB468 that overexpress EGFR and the BT474 and the SUM225 that overexpress HER2 were used for these experiments. ${ }^{17,18}$

shRNA inhibition of SHP2 and its effect on ERK1/2 and Akt activation. Cells expressing the SHP2 shRNA are referred to as shRNA, parental lines as PAR and cells expressing a scrambled control shRNA as CON. As shown in Figure 1a, expression of the SHP2 protein was inhibited by approximately $75 \%$ in the shRNA cells. Reblotting the membrane with anti- $\beta$-actin antibody indicated that comparable amount of total protein was loaded to each lane. These results suggest that SHP2 could be constitutively inhibited by shRNA, an approach that provides opportunities for studying the role of SHP2 in cell transformation and xenograft tumor growth.

The proficiency of the shRNA inhibition of SHP2 was confirmed by determining state of EGF-induced ERK $1 / 2$ and Akt activations. The EGF-induced ERK1/2 and Akt activation was suboptimal and short lived in shRNA, whereas it was sustained for about $4 \mathrm{~h}$ in the PAR and the CON cells of the BT20 and the BT474 line (Figure 1b). Reblotting the membranes with anti- $\beta$-actin or anti-ERK2 antibodies showed that there was a comparable amount of total protein in each lane. Similar results were obtained in the SUM225 and the MDA-MB468 cells (data not shown). Therefore, shRNA inhibition of SHP2 expression by approximately $75 \%$ was quite sufficient to abolish EGF-induced ERK1/2 and Akt activation.

Breast cancer cell transformation phenotypes are SHP2 dependent. The BT474 and the SUM225 cells typically grow as patchy, multilayered and aggregated colonies with less-defined intercellular boundaries, and they hardly go to
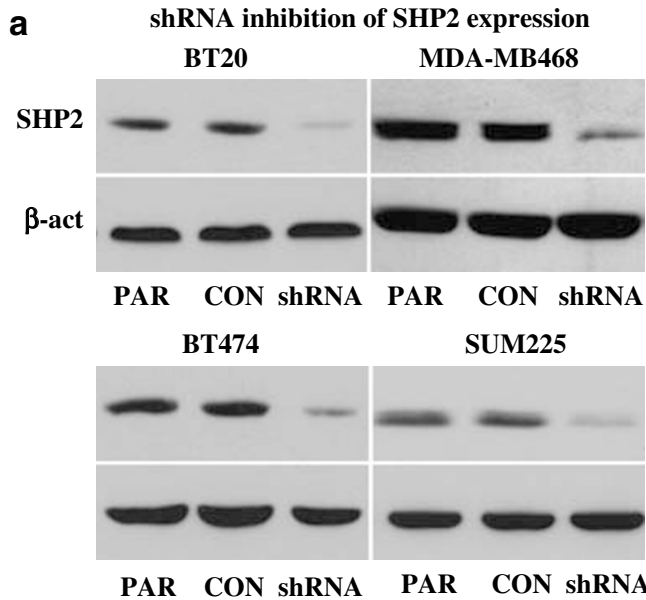

b

Analysis of ERK1/2 and Akt activation

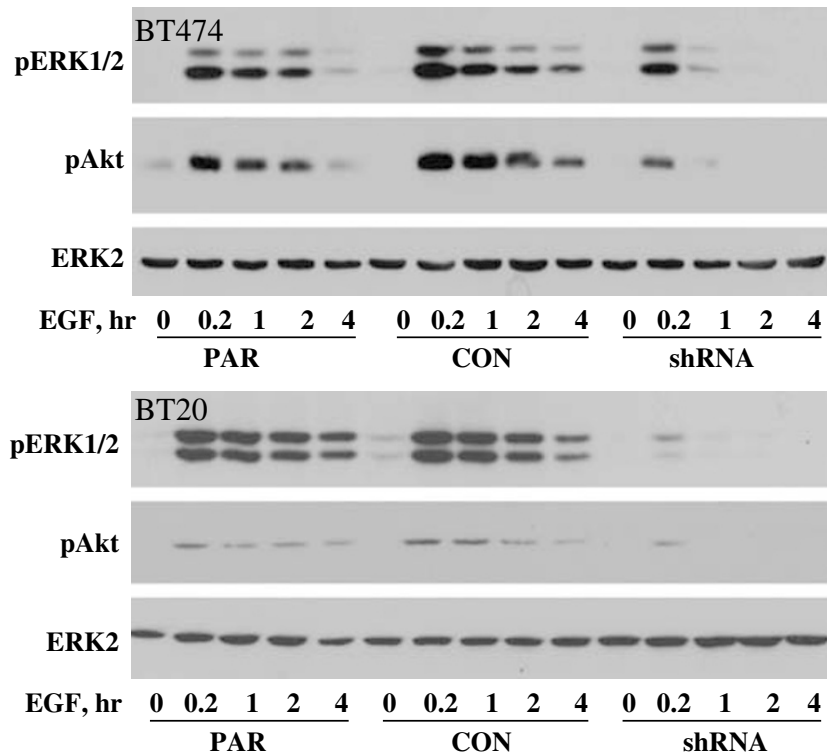

Figure 1 (a) Retrovirus-mediated expression of specific anti-Src homology phosphotyrosyl phosphatase 2 (SHP2) small hairpin RNA (shRNA) suppresses SHP2 protein expression by approximately $75 \%$ as determined by anti-SHP2 immunoblotting. (b) The PAR, the CON and shRNA cell derived from the BT474 and the BT20 lines were serum-starved for $\sim 12 \mathrm{~h}$ and then stimulated with $100 \mathrm{ng} / \mathrm{ml}$ EGF for the indicated time points. Lysates prepared from these cells were analyzed by immunoblotting with anti-phspho-ERK1/2 and anti-phospho-Akt polyclonal antibodies. $\beta$-actin was used as a loading control in (a), whereas ERK2 was used for the same purpose in (b). PAR, parental cells; CON, cells expressing control shRNA; shRNA, cells expressing the SHP2-specific shRNA

confluency. The MDA-MB468 cells are spindle shaped in morphology and thus grow as interspersed and networked jumble at high density. The BT20 cells also exhibit a spindleshaped morphology at low density with little intercellular adhesion, but form a sheet of elongated cells with islands of multilayered clumps at high density. Inhibition of SHP2 induced a dramatic change in the morphology and transformation phenotypes of all of the breast cancer cells tested in this study. As expected, the CON cells exhibited morphologies reminiscent of the parental PAR lines. In contrast, the shRNA cells grew as highly ordered monolayer 
sheet with clearly defined intercellular boundaries, flattened morphology and a characteristic polygonal shape typical of the cobblestone-like appearance of the MCF-10A, the immortalized breast epithelial cell line (Figure 2a). These results suggest that shRNA-mediated ablation of the SHP2
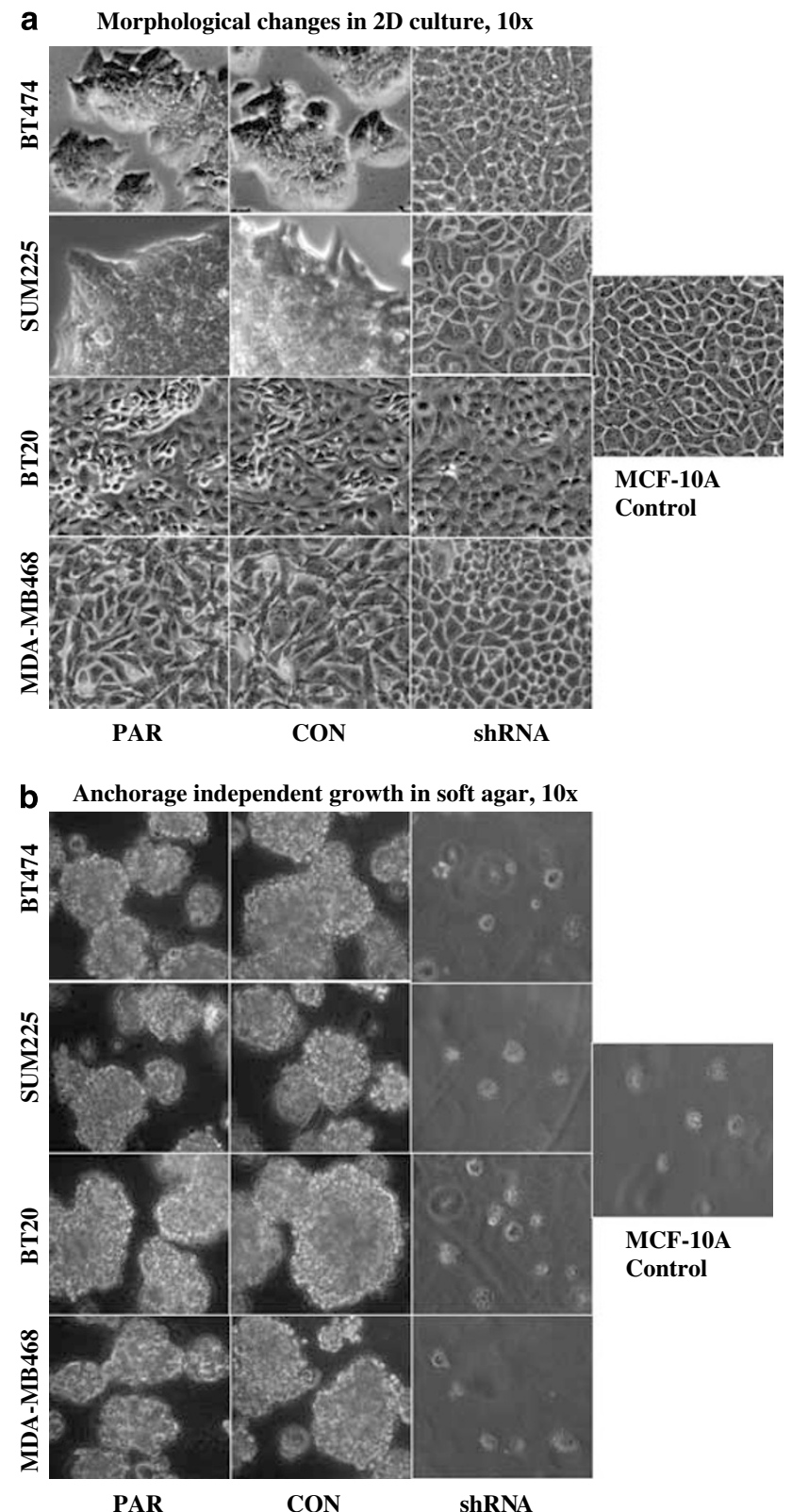

Figure 2 Small hairpin RNA (shRNA) inhibition of Src Homology Phosphotyrosyl phosphatase 2 (SHP2) reverses cell transformation. (a) The PAR, the CON and shRNA cells derived from the indicated four breast cancer cells were grown to confluency in culture dishes and phase contrast pictures were collected under Olympus IX70 microscope equipped with a digital camera. (b) Anchorageindependent growth assay in agar. Cells were seeded in agar as described in the materials and methods and their ability to form colonies was evaluated by observation under a microscope. Phase contrast pictures were taken after 7 days of incubation. The PAR and the CON cells formed large colonies, whereas the shRNA cells did not, suggesting that inhibition of SHP2 leads to loss of anchorageindependent growth. The MCF-10A cells were used as negative controls in both (a) and (b) protein induces reversion of breast cancer cells to a phenotype that resembles the normal breast epithelial cell morphology.

The four breast cancer cells tested here are proficient in anchorage-independent growth. ${ }^{19,20}$ To see if inhibition of SHP2 affects this phenotype, we seeded approximately $10^{4}$ cells from each line in soft agar and monitored colony formation by observation under a microscope. As shown in Figure $2 b$, the PAR and the CON cells formed large colonies in 7 days, whereas the shRNA cells could not. As expected, the MCF-10A did not form colonies in soft agar. Given that anchorage-independent growth is one of the traits of a cancer cell, the inability of the shRNA cells to form colonies demonstrates that SHP2 is required for the anchorageindependent growth property of breast cancer cells.

Inhibition of SHP2 induces a normal breast epithelial phenotype. The findings in Figure 2 were further corroborated by $3 \mathrm{D}$ matrigel assays, sometimes referred to as laminin-rich basement membrane (LRBM) culture. In this system, breast cancer cells continue to grow and form a disorganized mass of cells, whereas breast epithelial cell lines like the MCF-10A multiply a few times, stop growth, acquire a polarized morphology and form acini-like structures. ${ }^{21}$ Surprisingly, the shRNA cells formed acini-like structures reminiscent of the MCF-10A cells. In contrast, the PAR and the CON cells formed disorganized mass of cells (Figure 3a). Data shown was from the BT474 and the BT20 lines; similar results were obtained with the SUM225 and MDA-MB468 lines (not shown).

To further characterize the organizational architecture of cells in the matrigel, slides were co-stained with anti- $\alpha 6$ integrin antibody for cell polarity and anti-E-cadherin antibody for lateral cell-cell adhesion and then analyzed by immunofluorescence (IF) confocal microscopy as previously described. ${ }^{21,22}$ Similar to the MCF-10A cells, the shRNA cells formed polarized acini with vertical ECM-integrin and lateral cell-cell adhesion with hollow lumen (Figure 3b). On the other hand, the PAR and the CON cells formed nonpolarized, large and disorganized cellular mass with no hollow lumen. Microdissection imaging further confirmed that the shRNA cells formed spheroid structures with hollow lumen, whereas the PAR and CON cells were filled with cellular mass (Figure 3c). Data shown were from cells of BT20 and BT474 origin; similar results were obtained with the MDA-MB468 and SUM225 cells (not shown). By and large, these results demonstrate that SHP2 promotes the transformation phenotypes of breast cancer cells and that its inhibition leads to reversion to a normal breast epithelial phenotype.

SHP2 is required for cell migration and scattering. Previous work has shown that SHP2 suppresses cell adhesion and enhances motility. ${ }^{23-26} \mathrm{We}$ sough to investigate the significance of SHP2 inhibition on breast cancer cells motility and scattering. Initially, this possibility was tested by the monolayer wound-healing assay. The CON cells of the BT20 (Figure 4a, top panel) and the MDA-MB468 (not shown) lines migrated and filled the gap in less than $36 \mathrm{~h}$, whereas the corresponding shRNA cells were unable to do so even after $48 \mathrm{~h}$. Consistent with 
a Matrigel assay, Phase contrast, 10x

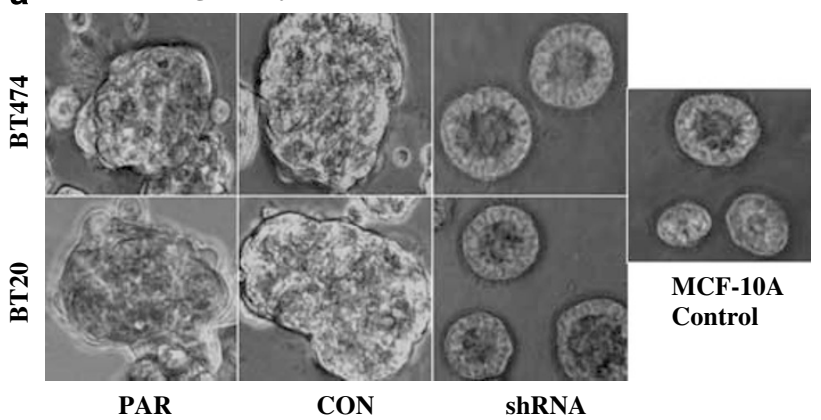

b Matrigel assay, Immunofluorescence, 10x
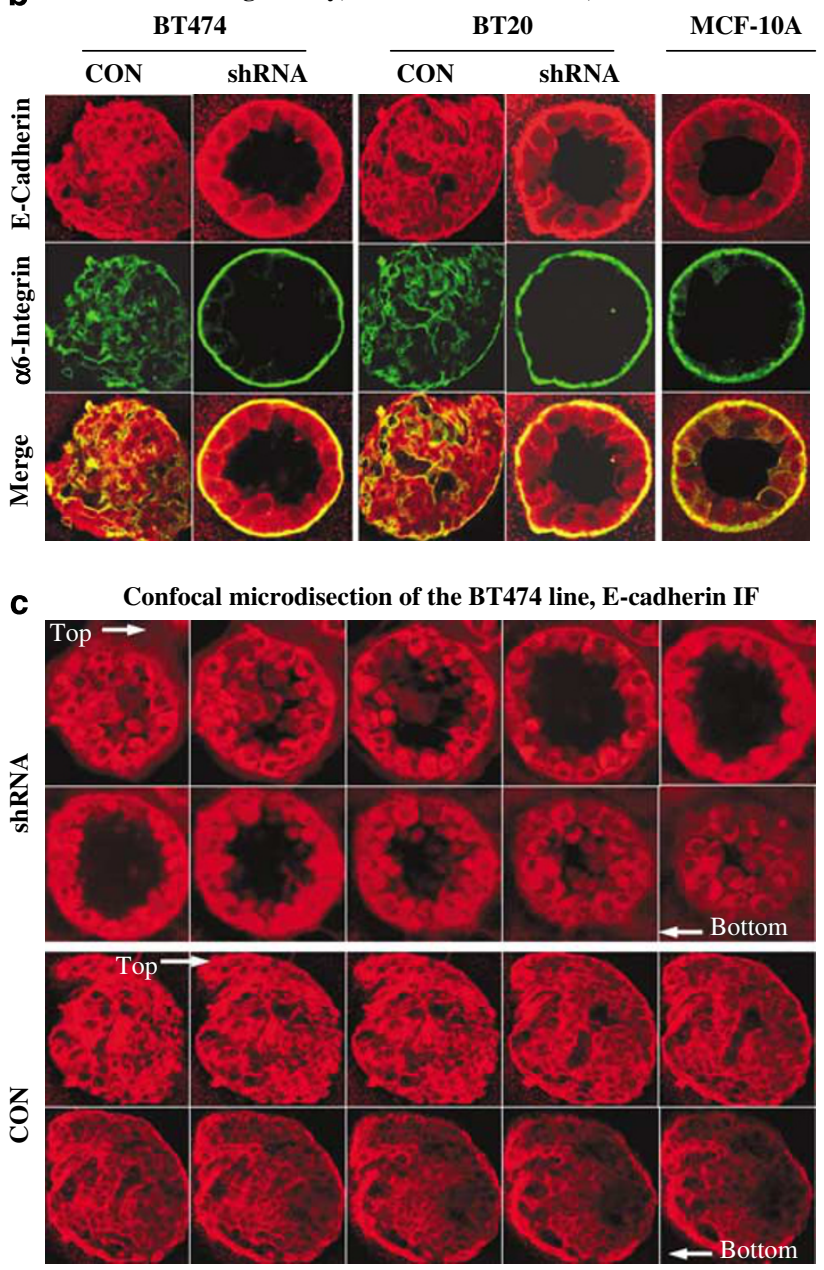

Figure 3 Small hairpin RNA (shRNA)-mediated ablation of the Src Homology Phosphotyrosyl phosphatase 2 (SHP2) protein induces differentiation of breast cancer cells to a normal phenotype. (a) Cells were seeded onto laminin-rich basement membrane (LRBM) also called matrigel and incubated for approximately 15 days before phase contrast pictures were taken. (b) Immunofluorescence staining of cells grown in LRBM 3D cultures. The shRNA cells formed polarized acini-like structures reminiscent of the MCF-10A cells, the positive controls for acini formation. The shRNA and the MCF-10A cell exhibited integrin-mediated vertical interaction with the basement membrane, adherens junction-mediated lateral cellcell interaction and a central hollow lumen. In contrast, the PAR and the CON cells formed disorganized cellular mass. (c) Comparative confocal slice images of the CON and the shRNA cells depicting the spheroidal structures with a hollow lumen in the shRNA cells and the absence of such a lumen, but amorphous cellular mass in the CON cells their compact growth property, the CON cells of the BT474 (Figure 4a, bottom panel) and the SUM225 (not shown) lines were slow in filling the gap, but performed significantly better than their shRNA counter parts. Nonetheless, the above results are consistent with a positive role for SHP2 in breast cancer cell migration.

The above results were further corroborated by cell scattering assay to see if the observed cell migrationpromoting effect of SHP2 was linked to its ability to modulate adherens junction-mediated cell-cell interaction. As expected, the CON cells of the BT20 line formed dispersed colonies with relatively low intercellular adhesion, whereas those from BT474 line formed highly aggregated colonies. On the other hand, the shRNA cells of both lines formed highly ordered sheet of colonies with defined intercellular boundaries (Figure 4b). Upon EGF stimulation, the CON cells of the BT20 line scattered further, whereas those of the BT474 line rather retracted, became elongated and tended to move as a group. In contrast, EGF stimulation did not cause any significant change in the shRNA cells. Cells derived from the MDA-MB468 line exhibited similar phenotypes to that of the BT20, whereas those from the SUM225 to the BT474 (data not shown). These cells were further analyzed by IF microscopy by co-staining with antibodies against the two major proteins involved in adherens junction, E-cadherin and $\beta$-catenin.

In conformity to phase contrast pictures, the CON cells of the BT20 line showed low E-cadherin and $\beta$-catenin staining at cell-cell contact sites, whereas those of the BT474 origin showed an intense staining. EGF stimulation induced dissolution of adherens junction, cell scattering and nuclear localization of $\beta$-catenin in the BT20 CON cells, but did not cause a similar change in the CON cells of BT474 line (Figure 4c). Rather, the BT474 CON cells exhibited change in colony shape reminiscent of group movement shown in the phase contrast pictures. On the other hand, the shRNA cells derived from both lines exhibited intense E-cadherin and $\beta$-catenin staining at cell-cell contact sites both in the absence and presence of EGF with no $\beta$-catenin translocation to the nucleus. As a result, colonies of shRNA cells remained virtually intact at least for $2 \mathrm{~h}$ following EGF stimulation. The results from the MDA-MB468 were similar to that of the BT20 line, whereas those from the AU565 were to the BT474 line (data not shown). Thus, the EGFR-overexpressing BT20 and MDA-MB468 breast cancer cells migrate individually, whereas the HER2-overexpressing BT474 and SUM225 cells move as a group. In either case, SHP2 is required for mediating cell scattering in EGFR and group movement in HER2-overexpressing breast cancer cells.

SHP2 is essential for EMT and its inhibition leads to MET. The dramatic difference in migratory behavior between the EGFR- and the HER2-overexpressing breast cancer cells led us to first investigate the expression state of E-cadherin, epithelial marker, and fibronectin and vimentin, mesenchymal markers. ${ }^{27-29}$ Considering the expression of these proteins in MCF-10A cells as normal, E-cadherin was downregulated, whereas fibronectin and vimentin were upregulated in the BT20 and in the MDA-MB468 cells. In contrast, E-cadherin was upregulated, whereas fibronectin and vimentin were virtually undetectable in the BT474 and 

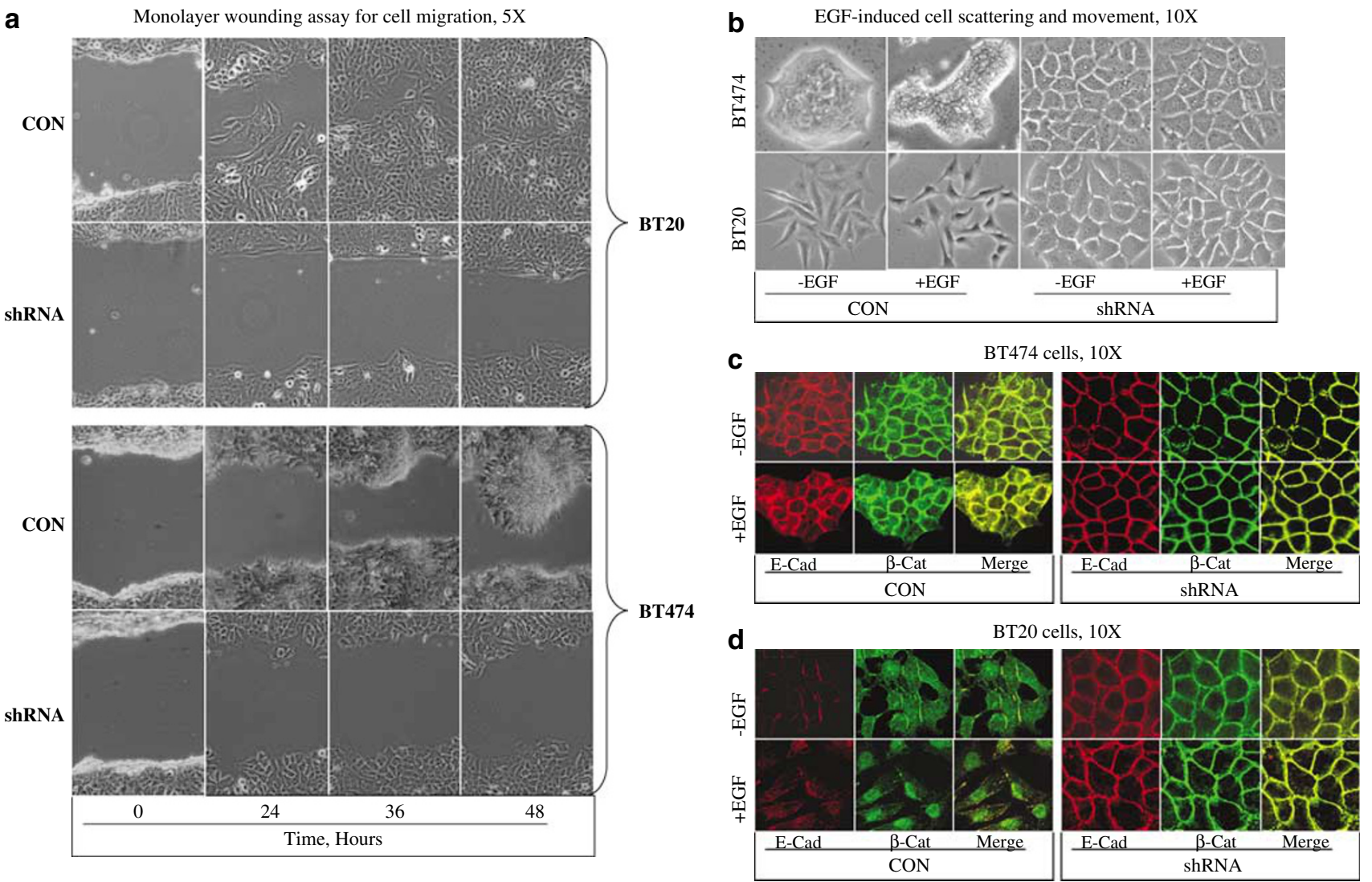

Figure 4 Effect of Src Homology Phosphotyrosyl phosphatase 2 (SHP2) inhibition on cell motility. (a) Monolayer wound-healing assay for testing the effect of SHP2 inhibition on cell migration. (b) Effect of SHP2 inhibition on EGF-induced cell scattering. Cells were stimulated with $100 \mathrm{ng} / \mathrm{ml}$ EGF for $2 \mathrm{~h}$ or left unstimulated, and phase contrast pictures were collected under Olympus IX71 microscope. (c) Confocal immunofluorescence images of the CON and the small hairpin RNA (shRNA) cells of the BT474 line. (d) Confocal immunofluorescence images of the CON and the shRNA cells of the BT20 line. Slides were double-stained with anti-E-cadherin anti- $\beta$-catenin antibodies and pictures were collected under Ziess scanning confocal microscope

SUM225 lines (Figure 5a). Band-density measurements showed that the BT20 and the MDA-MB-468 cells had $\sim 3-$ fold lower, whereas the BT474 and SUM225 cells had approximately 5-fold or more protein than the MCF-10A (Figure 5b). Similar band-density measurement of fibronectin and vimentin showed that the BT20 and the MDA-MB468 cells express at least five times more protein than the MCF10A cells, whereas the BT474 and the SUM225 cells have a very low amount barely detectable above background (Figure 5b). The low E-cadherin and elevated fibronectin and vimentin in the BT20 and the MDA-MB468 cells explain why these cells are fibroblastic and migratory, and why the vice versa is true in the BT474 and SUM225 cells. In addition, the elevated E-cadherin level in the BT474 and SUM225 cells explains why these cells grow as highly aggregated colonies and are unable to scatter upon growth factor stimulation.

Remarkably, shRNA inhibition of SHP2 restored 'normal' $\mathrm{E}$-cadherin and fibronectin levels. As shown in Figure $5 \mathrm{c}$, shRNA cells of the BT20 and the MDA-MB468 lines exhibited an increase, whereas shRNA cells of the BT474 and the SUM225 lines showed a decrease in E-cadherin expression, ultimately resulting in $\mathrm{E}$-cadherin levels that are comparable to the level found in MCF-10A cells. With regard to fibronectin, inhibition of SHP2 led to reduction in the BT20 and the MDA-
MB468 lines and an increase in the BT474 and the SUM225 lines, again leading to fibronectin levels comparable to MCF10A. Even more remarkable was that inhibition of SHP2 led to loss of vimentin expression by the BT20 and the MDA-MB468 cells, which had elevated levels (Figure $5 d$ ). Similar to the PAR and the CON counterparts, the shRNA cell of the BT474 and the SUM225 lines did not express vimentin. Reblotting with anti- $\beta$-actin antibody showed that total protein loaded to each lane was comparable. These results demonstrate that inhibition of SHP2 restores E-cadherin and fibronectin expression to levels found in the MCF-10A cells. In addition, they show that inhibition of SHP2 abolishes the expression of the mesenchymal marker vimentin by those breast cancer cells that had elevated levels. Therefore, SHP2 promotes epithelial to mesenchymal transition (EMT) and its inhibition leads to MET.

\section{Discussion}

Accumulating evidence suggests that SHP2 might play a pivotal role in cancer. First, SHP2 is a positive effector of

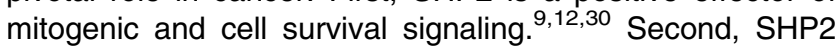
promotes cell transformation induced by $\mathrm{v}$-Src and the constitutively active form of FGFR3. ${ }^{1-3}$ Third, activating SHP2 mutations play causative role in Noonan Syndrome 

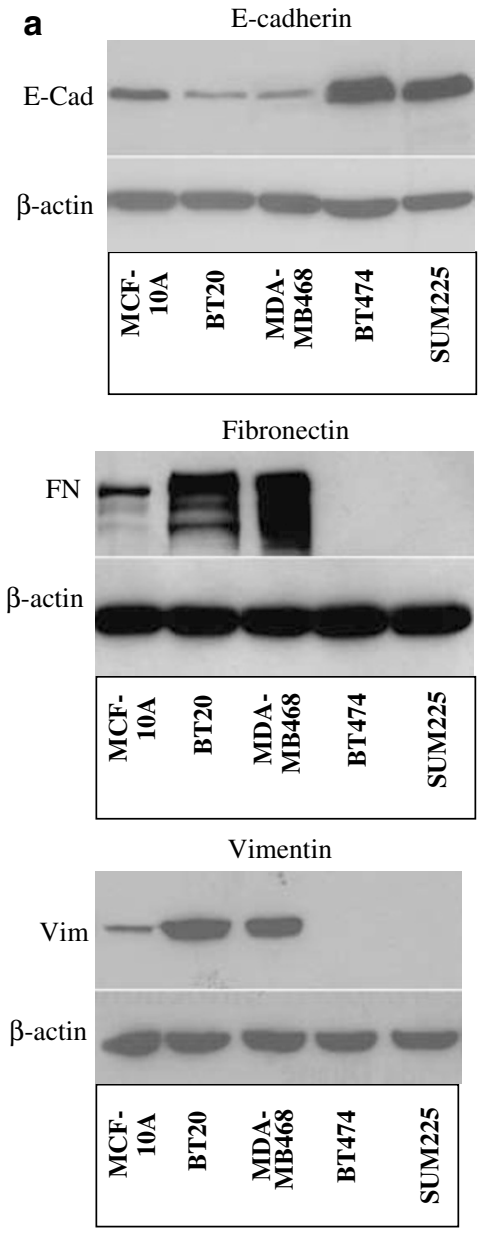

b Band density measurement

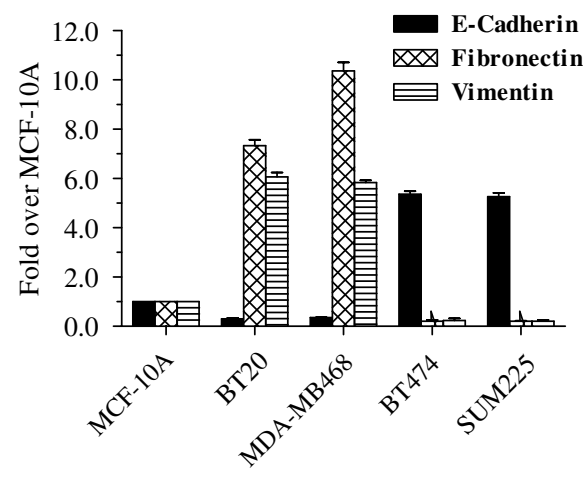

E-Cadherin and fibronectin

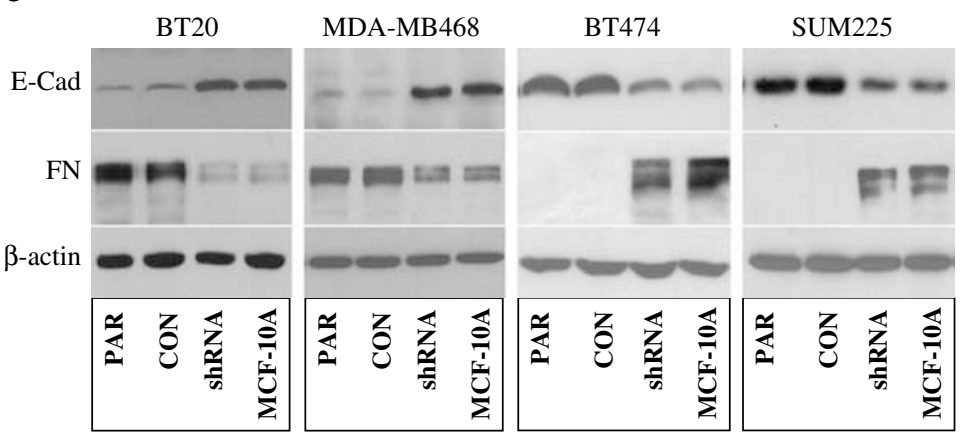

d

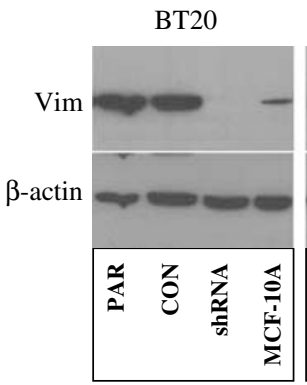

Vimentin

MDA-MB468

BT474

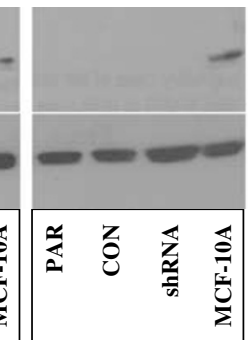

SUM225

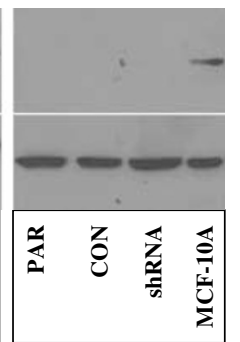

Figure 5 (a) Immunoblotting analysis of E-cadherin, the epithelial marker, and fibronectin and vimentin, the mesenchymal markers, in the four breast cancer cells used in this study. (b) Band-density measurement of E-cadherin, fibronectin and vimentin levels from three independent experiments. (c) Inhibition of Src Homology Phosphotyrosyl phosphatase 2 (SHP2) equilibrates E-cadherin and fibronectin levels to an amount found in MCF-10A cells. (d) Inhibition of SHP2 abrogates vimentin expression by the BT20 and the MDA-MB468 cells. Because the BT474 and the SUM225 cells do not normally express vimentin, the effect of SHP2 inhibition could not be determined

and associated leukemia. ${ }^{4}$ And finally, the SHP2 protein is overexpressed in approximately $70 \%$ of infiltrating ductal carcinoma of the human breast. ${ }^{16}$ On the basis of these premises, we sought to investigate the role of SHP2 in breast cancer cell transformation. In this report, we show that SHP2 is essential for breast cancer cell transformation and that its inhibition leads to reversion to a normal breast epithelial phenotype sometimes referred to as MET.

Inhibition of SHP2 suppresses EGF-induced ERK1/2 and Akt activation in breast cancer cell lines, suggesting that SHP2 is important for mitogenic and cell survival signaling not only in normal, but also in cancer cells. Furthermore, inhibition of SHP2 reverses breast cancer cell transformation as evidenced by acquisition of epithelial morphology, loss of anchorage-independent growth (Figure 2) and differentiation in 3D LRBM matrigel (Figure 3). Thus, SHP2 might play a pivotal role in breast cancer.

Remarkably, the highly fibroblastic BT20 and MDA-MB468 breast cancer cells lost their migratory property upon SHP2 inhibition. These results are consistent with previous reports that showed that SHP2 plays a positive role in fibroblast motility and migration. ${ }^{23-26}$ Thus, interference with SHP2 function might provide a means to inhibit cancer cell metastasis. Unexpectedly, we discovered that EGF can induce translocation of $\beta$-catenin to the nucleus in SHP2dependent manner. Although the mechanism is not clear, previous work has shown that tyrosine phosphorylation of $\beta$-catenin induces disengagement from adherens junctions, inhibits its degradation and promotes nuclear localization. ${ }^{31}$ It is possible that EGF induces $\beta$-catenin tyrosine phosphorylation, 
whereas at the same time activates SHP2 to dephosphorylate $\alpha$-catenin, ${ }^{3}$ the concerted action of which leads to $\beta$-catenin nuclear localization and transcriptional activation. Thus, $\beta$-catenin can be activated not only by the Wnt, but also by the EGFRR/HER2 signaling pathway.

The HER2-overexpressing BT474 and SUM225 cells are peculiar in their growth behavior; they grow as aggregated colonies with strong adherens junction-mediated intercellular adhesion. Upon EGF stimulation, the colonies of these cells shrink and tend to move as a group in SHP2-dependent manner. The strong adherens junction in these cells might explain why they grow as aggregated colonies. In this connection, it is intriguing to note that enhanced cell-cell adhesion can also contribute to cell transformation as these cells are highly tumorigenic in nude mice. Inhibition of SHP2 in these cells led to an epithelial morphology with vertical (cell-ECM) and lateral (cell-cell) interactions approximating those found in MCF-10A cells. Thus, SHP2 positively modulates divergent breast cancer cell phenotypes and its inhibition leads to reversion to a normal phenotype.

A large body of literature shows that downregulation of E-cadherin and upregulation of fibronectin and vimentin are associated with aggressive tumor growth, local invasion and metastasis to distant organs in many cancer types including breast cancer. ${ }^{32,33}$ However, our findings in four breast cancer cell lines, all isolated from infiltrating ductal carcinoma, exhibited divergent expression levels. The BT20 and the MDA-MB468 cells that overexpress EGFR have low E-cadherin and elevated fibronectin and vimentin, whereas the vice versa is true for the HER2 overexpressing BT474 and SUM225 cells; they have very low to undetectable fibronectin and vimentin (Figures $5 \mathrm{a}$ and $\mathrm{b}$ ). Consistent with previous reports, elevated expression of E-cadherin is inversely correlated to increased expression of fibronectin and vimentin. However, the occurrence of both events in a cell line-specific manner is somewhat unexpected. Given the reported tumor suppressor role of E-cadherin, its elevated expression in HER2-overexpressing breast cancer cells is puzzling as these cells are highly transformed, grow in soft agar and form tumors in nude mice. So far, there are no reports on elevated $\mathrm{E}$-cadherin expression in breast and other tumors. Similarly, given the highly tumorigenic phenotype of the BT474 and the SUM225 breast cancer cell lines, absence of vimentin expression is another unexpected observation; it indicates that vimentin is a marker for mesenchymal state, but not for cell transformation. It will be interesting to address the significance of an elevated E-cadherin and downregulated vimentin in cancer in future studies.

The most striking finding was that inhibition of SHP2induced equilibration in the expression levels of E-cadherin and fibronectin (Figure $5 \mathrm{c}$ ). Inhibition of SHP2 led to an increase in E-cadherin in the BT20 and the MDA-MB468 cells that had low levels and a decrease in the BT474 and SUM225 cells that had elevated levels, ultimately balancing to an amount found in the MCF-10A cells. Similarly, inhibition of SHP2 led to a reduction in fibronectin in the BT20 and the MDA-MB468 cells that had elevated levels and an acquisition by the BT474 and the SUM225 cells that had undetectable levels. With regard to vimentin, inhibition of SHP2 led to loss of vimentin expression by the BT20 and the MDA-MB468 cells, but did not lead to derepression in the BT474 and the SUM225 cells (Figure $5 \mathrm{~d}$ ), suggesting that vimentin is not required for normal epithelial homeostasis. Several conclusions can be drawn from these findings. First, physiological levels of both fibronectin and E-cadherin are important for maintenance of normal breast epithelial phenotype. Second, not only downregulation and mutational inactivation of $\mathrm{E}$-cadherin, but also its elevated expression might contribute to cell transformation and tumor growth. And third, elevated expression of vimentin predicts the mesenchymal, but not its transformation state. Thus, one of the mechanisms by which SHP2 promotes breast cancer cells transformation is by modulating the expression of E-cadherin, fibronectin and vimentin.

The molecular mechanism of SHP2 in regulating diverse signaling pathways and cellular processes is just beginning to be elucidated. We have previously demonstrated that SHP2 promotes Ras activation by blocking RasGAP recruitment to the plasma membrane. ${ }^{9}$ Others have shown that SHP2 promotes Ras and ERK1/2 activation by facilitating RTKinduced activation of the SFKs. ${ }^{12}$ SHP2 also promotes $\beta 1$ integrin-induced SFK activation in a similar way, leading to Ras and ERK1/2 activation. ${ }^{34}$ The Gab1-SHP2 signaling axis downstream of RTKs has also been shown to be critical for growth factor-induced signaling. ${ }^{35}$ The discovery of Gab2 ${ }^{36}$ and SHP2 ${ }^{16}$ overexpression in primary breast tumors and their cooperation in promoting HER2-induced mammary tumorigenesis in transgenic mice ${ }^{37}$ further highlight the critical role SHP2 plays in breast cancer. In conclusion, promotion of cell transformation by SHP2 is a multifaceted process that involves, at least in part, augmentation of cell proliferation and survival and suppression of adherens junction-mediated cellcell interaction. Therefore, inhibition of SHP2 might provide a means for treating breast cancer as it could lead to MET.

\section{Materials and Methods}

Cells, cell culture and reagents. The breast cancer cell lines used in this study were the BT20, MDA-MB468, BT474 and SUM225. The MCF-10A, the immortalized breast epithelial cell line, was used as a control. The breast cancer cell lines were purchased from ATCC and Asterand Inc., whereas the MCF-10A cells were obtained from Dr. Daniel Flynn (Marry Babb Randolph Cancer Center, West Virginia University). The BT474 cells were grown in RPMI 1640, the SUM225 in F12, whereas the BT20 and the MDA-MB468 were in Dulbecco's modified Eagle's medium (DMEM) all supplemented with $10 \%$ fetal calf serum. The MCF-10A were grown in DMEM supplemented with $5 \%$ horse serum, $10 \mu \mathrm{g} / \mathrm{ml}$ recombinant human insulin, $0.5 \mu \mathrm{g} / \mathrm{ml}$ hydrocortisone, $20 \mathrm{ng} / \mathrm{ml} \mathrm{EGF} \mathrm{(PeproTech)} \mathrm{and} 100 \mathrm{ng} / \mathrm{ml}$ cholera toxin (Sigma), as previously described. ${ }^{21}$ The MDA-MB468 cells were maintained at $37^{\circ} \mathrm{C}$ in the absence of $\mathrm{CO}_{2}$, whereas the rest were maintained at the same temperature, but with $5 \% \mathrm{CO}_{2}$. The anti- $\beta$-catenin, anti-E-cadherin and antifibronectin antibodies were from Santa Cruz, anti- $\beta$-actin antibody was from Sigma, and anti-PTP1D (SHP2) and anti-vimentin antibodies were from Pharmagen. The anti-phospho-ERK1/2 and anti-phospho-Akt antibodies were from Cell Signaling. Horseradish peroxidase-conjugated secondary antibodies were purchased from Amersham, whereas Alexa fluor 488 and rhodamine-labeled secondary antibodies were from Molecular Probes.

shRNA construction and production of stable cell lines. shRNAmediated ablation of the expression of the SHP2 protein was accomplished by retroviral transduction (BD Biosciences, Palo Alto, CA, USA). A short doublestranded DNA oligonucleotide that codes for anti-SHP2 shRNA was custom synthesized (Integrated DNA Technologies) and ligated into the BamHI and EcoRI sites of the retroviral vector termed PSIREN-RetroQ-TetP. The targeting oligonucleotide sequence was $5^{\prime}$-GAAATGGAGCTGTCACCCA-3'. Although the pSIREN-RetroQ-TetP vector can be used as a tetracycline-inducible system in 
conjunction with $\mathrm{pQC}$-tTS-IN that express the Tet-responsive repressor protein, it can also be used alone to direct constitutive expression of inserted shRNA. We chose the latter for our experiments as an approximately $70 \%$ reduction in SHP2 protein does not lead to cell death, but to differentiation. The SHP2 shRNA construct was transfected into appropriate packaging cells and, after $48 \mathrm{~h}$, transient supernatants were used for infecting the four breast cancer cell lines mentioned above. , Cells were treated with puromycin to remove noninfected cells $48 \mathrm{~h}$ postinfection. Because puromycin-resistant populations exhibited heterogeneity in cellular response, cells that showed maximum SHP2 inhibition, as determined by anti-SHP2 immunoblotting, were obtained by cloning.

Preparation of cell lysates and immunoblotting analysis. Cells were lyzed in lysis buffer containing $20 \mathrm{~mm}$ Tris- $\mathrm{HCl}, \mathrm{pH} 7.4,150 \mathrm{~mm} \mathrm{NaCl}, 50 \mathrm{~mm}$ $\mathrm{NaF}, 1 \mathrm{~mm}$ EDTA, $10 \%$ glycerol, $1 \%$ triton $\mathrm{X}-100,1 \mathrm{~mm}$ Na orthovanadate and a protease inhibitor cocktail. All lysates were briefly sonicated to break chromosomal DNA that could interfere with gel separation of proteins. After clearing by centrifugation, the lysates were analyzed as described in the respective experiments. For electrophoresis gel separation of proteins, the cell lysates were denatured by boiling with Laemmli sample buffer and run on an $8 \%$ denaturing polyacrylamide gel. After transfer onto a nitrocellulose membrane and blocking with $3 \%$ bovine serum albumin, the membranes were stained with the primary antibody either overnight at $4^{\circ} \mathrm{C}$ or for $2 \mathrm{~h}$ at room temperature. Staining with the secondary antibody was for $1 \mathrm{~h}$ at room temperature. We used the chemiluminescence detection method for all immunoblotting experiments.

Anchorage-independent growth in agar. The ability of cells to form colonies in soft agar was performed as previously described. ${ }^{38}$ Briefly, $6 \mathrm{~cm}$ cell culture plates were overlaid with $0.3 \%$ agar in the growth medium and allowed to solidify. Cells were suspended in $3 \mathrm{ml}$ of growth medium and mixed with melted agar to a final concentration of $0.3 \%$ and immediately poured onto the agar overlay. After $5 \mathrm{~min}$ of incubation at room temperature for the agar to solidify, the cells were transferred to a $37^{\circ} \mathrm{C}$ and $5 \% \mathrm{CO}_{2}$ incubator for 7 days. During this time, the cells were fed with soft agar medium every 3 days. Colony formation was visualized under a microscope and phase contrast pictures were taken using an Olympus IX71 microscope equipped with Olympus DP30BW digital camera.

Cell differentiation in LRBM cultures. LRBM cultures were performed as previously described. ${ }^{21}$ Briefly, $80 \mu$ l of growth factor-reduced LRBM medium (BD Biosciences) was overlaid onto four-well chamber slides (Falcon) and allowed to solidify for $15 \mathrm{~min}$ at $37^{\circ} \mathrm{C}$ in a cell culture incubator. Approximately $10^{3}$ cells resuspended in $250 \mu \mathrm{l}$ of assay medium (DMEM supplemented with $2 \%$ horse serum, $10 \mu \mathrm{g} / \mathrm{ml}$ recombinant human insulin, $0.5 \mu \mathrm{g} / \mathrm{ml}$ hydrocortisone, $5 \mathrm{ng} / \mathrm{m}$ EGF, $100 \mathrm{ng} / \mathrm{ml}$ cholera toxin and $1 \times$ penicillin/streptomycin) were plated on a solidified LRBM and cultured at $37^{\circ} \mathrm{C}$ in $7 \% \mathrm{CO}_{2}$ incubator. The cells were refed with assay medium every 4 days and phase contrast pictures were taken after 15 days. The same cultures were processed for confocal IF microscopy as previously described. ${ }^{21,39} \mathrm{~A}$ brief description of IF protocol used for both $2 \mathrm{D}$ and $3 \mathrm{D}$ cultures is given below.

Monolayer wound-healing assay. Cells were grown to confluency, wounded by scratching with a pipette tip, and then incubated for a total of $48 \mathrm{~h}$. Phase contrast pictures were taken at $0,24,36$ and $48 \mathrm{~h}$ time points following wounding to see if inhibition of SHP2 interferes with the ability of cells to migrate and fill the newly created space.

Immunofluorescence microscopy. Cell in both LRBM chambers and 2D cultures (grown on coverslips) were fixed with $4 \%$ paraformaldehyde for $20 \mathrm{~min}$ washed three times with PBS (5 min each), permeablized with $0.2 \%$ triton X-100 in PBS for $30 \mathrm{~min}$ and blocked with $3 \%$ bovine serum albumin in PBS for $1 \mathrm{~h}$. The samples were then stained with primary antibodies at $4^{\circ} \mathrm{C}$ overnight, washed three times with PBS containing $0.2 \%$ triton X-100 and incubated with fluorescent-labeled secondary antibodies for $1 \mathrm{~h}$ at room temperature. After washing three times, the coverslips were mounted onto microscopic slides, whereas the LRBM slides were covered with appropriate size coverslips after removal of chambers. Finally, slides were scanned with laser scanning microscope (LSM 510, Zeiss) and pictures collected.
Acknowledgements. We thank Dr Daniel Flynn from the Marry Babb Randolph Cancer Center, West Virginia University for providing the MCF-10A cell line used in this study. This publication was made possible by grant number CA124940 from National Cancer Institute ( $\mathrm{NCl})$, component of the National Institute of Health $(\mathrm{NIH})$ to YMA.

1. Hakak Y, Hsu YS, Martin GS. Shp-2 mediates v-Src-induced morphological changes and activation of the anti-apoptotic protein kinase Akt. Oncogene 2000; 19: 3164-3171.

2. Agazie YM, Movilla N, Ischenko I, Hayman MJ. The phosphotyrosine phosphatase SHP2 is a critical mediator of transformation induced by the oncogenic fibroblast growth factor receptor 3. Oncogene 2003; 22: 6909-6918.

3. Burks J, Agazie YM. Modulation of alpha-catenin Tyr phosphorylation by SHP2 positively effects cell transformation induced by the constitutively active FGFR3. Oncogene 2006; 25: 7166-7179.

4. Tartaglia M, Kalidas K, Shaw A, Song X, Musat DL, van der Burgt I et al. PTPN11 mutations in Noonan syndrome: molecular spectrum, genotype-phenotype correlation, and phenotypic heterogeneity. Am J Hum Genet 2002; 70: 1555-1563.

5. Araki T, Mohi MG, Ismat FA, Bronson RT, Williams IR, Kutok JL et al. Mouse model of Noonan syndrome reveals cell type- and gene dosage-dependent effects of Ptpn11 mutation. Nat Med 2004; 10: 849-857.

6. Mohi MG, Williams IR, Dearolf CR, Chan G, Kutok JL, Cohen S et al. Prognostic, therapeutic, and mechanistic implications of a mouse model of leukemia evoked by Shp2 (PTPN11) mutations. Cancer Cell 2005; 7: 179-191.

7. Feng GS, Hui CC, Pawson T. SH2-containing phosphotyrosine phosphatase as a target of protein-tyrosine kinases. Science 1993; 259: 1607-1611.

8. Frearson JA, Alexander DR. The phosphotyrosine phosphatase SHP-2 participates in a multimeric signaling complex and regulates T cell receptor (TCR) coupling to the Ras/ mitogen-activated protein kinase (MAPK) pathway in Jurkat T cells. J Exp Med 1998; 187: $1417-1426$.

9. Agazie YM, Hayman MJ. Molecular mechanism for a role of SHP2 in epidermal growth factor receptor signaling. Mol Cell Biol 2003; 23: 7875-7886.

10. Cleghon V, Feldmann P, Ghiglione C, Copeland TD, Perrimon N, Hughes DA et al. Opposing actions of CSW and RasGAP modulate the strength of Torso RTK signaling in the Drosophila terminal pathway. Mol Cell 1998; 2: 719-727.

11. Montagner A, Yart A, Dance M, Perret B, Salles JP, Raynal P. A novel role for Gab1 and SHP2 in epidermal growth factor-induced Ras activation. J Biol Chem 2005; 280: 5350 5360 .

12. Zhang SQ, Yang W, Kontaridis MI, Bivona TG, Wen G, Araki T et al. Shp2 regulates SRC family kinase activity and Ras/Erk activation by controlling Csk recruitment. Mol Cell 2004; 13: $341-355$

13. Saxton TM, Henkemeyer M, Gasca S, Shen R, Rossi DJ, Shalaby F et al. Abnormal mesoderm patterning in mouse embryos mutant for the $\mathrm{SH} 2$ tyrosine phosphatase Shp-2. EMBO J 1997; 16: 2352-2364.

14. Araki $T$, Nawa $H$, Neel BG. Tyrosyl phosphorylation of Shp2 is required for normal ERK activation in response to some, but not all, growth factors. J Biol Chem 2003; 278: 41677-41684.

15. Hof P, Pluskey S, Dhe-Paganon S, Eck MJ, Shoelson SE. Crystal structure of the tyrosine phosphatase SHP-2. Cell 1998; 92: 441-450.

16. Zhou X, Coad J, Ducatman B, Agazie YM. SHP2 is upregulated in breast cancer cells and in infiltrating ductal carcinoma of the breast, implying its involvement in breast oncogenesis. Histopathology 2008 (in press)

17. Gensler M, Buschbeck M, Ullrich A. Negative regulation of HER2 signaling by the PESTtype protein-tyrosine phosphatase BDP1. J Biol Chem 2004; 279: 12110-12116.

18. Konecny GE, Pegram MD, Venkatesan N, Finn R, Yang G, Rahmeh M et al. Activity of the dual kinase inhibitor lapatinib (GW572016) against HER-2-overexpressing and trastuzumab-treated breast cancer cells. Cancer Res 2006; 66: 1630-1639.

19. Knuefermann C, Lu Y, Liu B, Jin W, Liang K, Wu L et al. HER2/PI-3K/Akt activation leads to a multidrug resistance in human breast adenocarcinoma cells. Oncogene 2003; 22: 3205-3212.

20. Ono M, Kuwano M. Molecular mechanisms of epidermal growth factor receptor (EGFR) activation and response to gefitinib and other EGFR-targeting drugs. Clin Cancer Res 2006; 12: 7242-7251.

21. Debnath J, Muthuswamy SK, Brugge JS. Morphogenesis and oncogenesis of MCF-10A mammary epithelial acini grown in three-dimensional basement membrane cultures. Methods 2003; 30: 256-268.

22. Bissell MJ, Radisky DC, Rizki A, Weaver VM, Petersen OW. The organizing principle: microenvironmental influences in the normal and malignant breast. Differentiation 2002; 70: $537-546$.

23. Inagaki K, Noguchi T, Matozaki T, Horikawa T, Fukunaga K, Tsuda M et al. Roles for the protein tyrosine phosphatase SHP-2 in cytoskeletal organization, cell adhesion and cell migration revealed by overexpression of a dominant negative mutant. Oncogene 2000; 19: 75-84.

24. Kodama A, Matozaki T, Fukuhara A, Kikyo M, Ichihashi M, Takai Y. Involvement of an SHP-2-Rho small G protein pathway in hepatocyte growth factor/scatter factor-induced cell scattering. Mol Biol Cell 2000; 11: 2565-2575. 
25. Manes S, Mira E, Gomez-Mouton C, Zhao ZJ, Lacalle RA, Martínez-A C. Concerted activity of tyrosine phosphatase SHP-2 and focal adhesion kinase in regulation of cell motility. Mol Cell Biol 1999; 19: 3125-3135.

26. Yu DH, Qu CK, Henegariu O, Lu X, Feng GS. Protein-tyrosine phosphatase Shp-2 regulates cell spreading, migration, and focal adhesion. J Biol Chem 1998; 273: 21125-21131.

27. Ahmed N, Maines-Bandiera S, Quinn MA, Quinn MA, Unger WG, Dedhar S et al. Molecular pathways regulating EGF-induced epithelio-mesenchymal transition in human ovarian surface epithelium. Am J Physiol Cell Physiol 2006; 290: C1532-C1542.

28. Huber MA, Kraut N, Beug $\mathrm{H}$. Molecular requirements for epithelial-mesenchymal transition during tumor progression. Curr Opin Cell Biol 2005; 17: 548-558.

29. Zhou BP, Deng J, Xia W, Xu J, Li YM, Gunduz M et al. Dual regulation of Snail by GSK 3beta-mediated phosphorylation in control of epithelial-mesenchymal transition. Nat Cell Biol 2004; 6: 931-940.

30. Oh ES, Gu H, Saxton TM, Timms JF, Hausdorff S, Frevert EU et al. Regulation of early events in integrin signaling by protein tyrosine phosphatase SHP-2. Mol Cell Biol 1999; 19: 3205-3215.

31. Hu P, O'Keefe EJ, Rubenstein DS. Tyrosine phosphorylation of human keratinocyte betacatenin and plakoglobin reversibly regulates their binding to E-cadherin and alpha-catenin J Invest Dermatol 2001; 117: 1059-1067.

32. Hajra KM, Fearon ER. Cadherin and catenin alterations in human cancer. Genes Chromosomes Cancer 2002; 34: 255-268.
33. Pierceall WE, Woodard AS, Morrow JS, Rimm D, Fearon ER. Frequent alterations in $\mathrm{E}$-cadherin and alpha- and beta-catenin expression in human breast cancer cell lines. Oncogene 1995; 11: 1319-1326.

34. Bertotti A, Comoglio PM, Trusolino L. Beta4 integrin activates a Shp2-Src signaling pathway that sustains HGF-induced anchorage-independent growth. J Cell Biol 2006; 175 993-1003.

35. Maroun CR, Naujokas MA, Holgado-Madruga M, Wong AJ, Park M. The tyrosine phosphatase SHP-2 is required for sustained activation of extracellular signal-regulated kinase and epithelial morphogenesis downstream from the met receptor tyrosine kinase. Mol Cell Biol 2000; 20: 8513-8525.

36. Daly RJ, Gu H, Parmar J, Lyons RJ, Kairouz R, Head DR et al. The docking protein Gab2 is overexpressed and estrogen regulated in human breast cancer. Oncogene 2002; 21: 5175-5181

37. Bentires-Alj M, Paez JG, David FS, Keilhack H, Halmos B, Naoki K et al. Activating mutations of the Noonan syndrome-associated SHP2/PTPN11 gene in human solid tumors and adult acute myelogenous leukemia. Cancer Res 2004; 64: 8816-8820.

38. Agazie $Y$, Ischenko I, Hayman M. Concomitant activation of the PI3K-Akt and the RasERK signaling pathways is essential for transformation by the V-SEA tyrosine kinase oncogene. Oncogene 2002; 21: 697-707.

39. Shaw KR, Wrobel CN, Brugge JS. Use of three-dimensional basement membrane cultures to model oncogene-induced changes in mammary epithelial morphogenesis. J Mammary Gland Biol Neoplasia 2004; 9: 297-310. 